\title{
Análisis clínico de 5 casos de intoxicación alcohólica en niños menores de 10 años
}

\author{
Dra. Ana María Vinet R. ${ }^{2}$; Dra, Pamela Oliva N.2
}

Alcohol intoxication in children younger than 10 year.

A review of 5 cases

\begin{abstract}
Five cases of alcohol intoxication in children less than 10 years old, discharged from the Hospital of Temuco between 1984 to 1986 are reviewed. Mast of the patients were male and of rural residence. Aicoholic beverages were given with parental consent. The main chinical tindings. were consciousness impaiment, hypertonia, trismus and seizures. The latter condition was sclated to hipoglycaemia in one patient. In younger patient recovery to neurologic normality was slower. Our study emphasizes the need of educational efforts addressed to show the risks of alcohol ingestion in children. Physicians must consider this diagnosis when facing young patients with consciousness impairnent.
\end{abstract}

(Key words: alcohol, intoxication, symptoms, (liagnosis).

La Novena Región de la Araucanía es un área de alto índice de consumo de alcohol en el pais, lo que está relacionado con un patrón cultural permisivo ${ }^{1}$ en la población general. Se ha demostrado que los hijos de padres bebedores consumen con mayor frecuencia alcohol en edades tempranas de la vida comparados con hijos de padres abstemios. ${ }^{2}$ No contamos con cifras regionales, pero estudios realizados en el área central de Santiago, muestran que en una población escolar el $27 \%$ ha ingerido alcohol antes de los 10 años de edad. Según Montenegro en estratos socioeconómicos medios y bajos, la ingesta de

1. Pediatra Hospital Regional de Temuco. Docente Depto. de Pediatría, Escuela de Medicina. Universidad de La Frontera.

2. Becada de Pediatría. Hospital Regional de Temuco. Universidad de La Frontera. alcohol comienza muchas veces en el período de recién nacido y a los 2 años el $90 \%$ de los niños pertenecientes a estos grupos han probado alguna vez este tipo de bebida. ${ }^{1}$

El niño tiene una gran susceptibilidad a los efectos del alcolool que le causan rápido y grave compromiso neurológico y metabólico, hipoglicemia e hipoxia que puede dejar secuelas en el sistema nervioso. ${ }^{3-7}$

En los casos de menores que se hospitalizan con compromiso neurológico de origen no preci. sado, rara vez se sospecha la intoxicación alcohólica, por lo que no se busca el antecedente de ingestión ni se completa el estudio metabólico. Esta falla en el diagnóstico se describe también en la literatura, ${ }^{7}$ y se puede relacionar además con la mayor velocidad con que es metabolizado el aloohol demostrada en los niños que duplica aproximadamente la del aduIto, ${ }^{8}$ obligando a 
sospechar temprano la posibilidad bajo pena de no alcanzar a detectar el alcohol en la sangre.

En este estudio se describen algunas caracteristicas clinicas y sociodemográficas del problema en nuestro ámbito.

\section{MATERIAL Y METODO}

Se buscaron retrospectivamente los casos de niños menores de 10 años egresados, entre agosto de 1984 y julio de 1986 del Servicio de Pediatria del Hospital Regional de Temucho, con diagnóstico de intoxicación aloohólica, sin afecciones neurológicas previas. Sc registraton 5 pacientes que corresponden a $0,3 \%$ de los egresos del Servicio de niños entre 2 y 10 años en el período.

En ellos se estudjaron la edad, scxo, procedencía, el tipo y cantidad de bebida ingerida, la provisión de ésta, el cstado de nutrición, cuadro clínico, compromiso reurológico, estudio de laboratorio y evolución. Las alcoholemias fueron medidas en el laboratorio del Hospital con el método de Widmarck.

\section{RESULTADOS}

Cuatro pacientes eran varones. Dos niños te- nían entre 2 y 3 años, 2 entre 4 y 5 años, 1 entre 7 y 8 años; 4 procedian de localidades rurales de la Región y 1 de la ciudad de Temuco. La bebida más ingerida fue el vino en cantidad variable $y$ fue administrado en cuatro de los casos con consentimiento de los padres o responsables legales del niño o por ellos mismos. En dos menores no se obtuvo el antecedente de ingesta alcohólica al ingresar al hospital.

En la evaluación neurológica inicial un paciente ingresó lúcido y orientado pero con alteración del equilibrio y la coordinación. Los otros cuatro presentaron compromiso de conciencia que fluctuó entre sopor superficial y coma, hipertonia generalizada y trismus (signo que hizo sospechar el diagnóstico en uno), reflejos osteotendineos aumentados y fondo de ojo normal. Con el curso de la evolución tres pacientes presentaron convulsiones (dos crisis tónico-clonicas generalizadas en cada caso) (tabla 1 ).

En el examen físico general y segmentario todos mostraban poliprea, uno piel enrojecida $y$ dos signos de compromiso pulmonar (uno neu-

Tabla 1.

Evaluación neurológica de 5 casos de intoxicación alcohólica en niṽos menores de 10 años

\begin{tabular}{|c|c|c|c|c|c|}
\hline Paciente & 1 & 2 & 3 & 4 & 5 \\
\hline Edad & 2 a $4 m$ & $2 a 11 \mathrm{~m}$ & $\overline{4}$ a $2 \mathrm{~m}$ & $4.6 \mathrm{~m}$ & 7 aก̃os \\
\hline $\begin{array}{l}\text { Compromiso } \\
\text { Conciencia }\end{array}$ & $\begin{array}{l}\text { Sopor } \\
\text { Superficial }\end{array}$ & $\begin{array}{l}\text { Sopor } \\
\text { Protundo }\end{array}$ & Lúcido & $\begin{array}{l}\text { Sopor } \\
\text { Medinno }\end{array}$ & Coma \\
\hline $\begin{array}{l}\text { Parex } \\
\text { Crancanos }\end{array}$ & Norimales & $\begin{array}{l}\text { Mlidriasis } \\
\text { con RFM (+) }\end{array}$ & Normales & Normales & Normales \\
\hline $\begin{array}{l}\text { Tono } \\
\text { Muscular }\end{array}$ & Hiperton 1́a & Hipertonis & Normal & Hipertonía & Hipertonia \\
\hline Trismus & Presente & Presente & Ausente & Presetute & Presente \\
\hline ROI & Normales & $\begin{array}{l}\text { Aumentidos } \\
\text { Clonus }\end{array}$ & Disminuidos & Aumentad'or & Aumentados \\
\hline $\begin{array}{l}\text { Resputsta } \\
\text { Plantar }\end{array}$ & Normal & Indiferente & Normal & Babusksy & Indiferente \\
\hline $\begin{array}{l}\text { Equilibrios y } \\
\text { Coordinacion }\end{array}$ & $\begin{array}{l}\text { No } \\
\text { cvaluable: }\end{array}$ & $\begin{array}{l}\text { No } \\
\text { cviluable }\end{array}$ & Alterads & $\begin{array}{l}\text { No } \\
\text { evaluable }\end{array}$ & $\begin{array}{l}\text { No } \\
\text { evaluable }\end{array}$ \\
\hline $\begin{array}{l}\text { Signus } \\
\text { Meningeos }\end{array}$ & Ausentes & Ausentes & Auscotes & Presentes & Ausentes \\
\hline Fondo Ojo & Normal & Normal & Normal & Norma! & Normal \\
\hline Cunvulsiones & Si & Si & No & No & Si \\
\hline
\end{tabular}

RFM: Reflejo fotomotor 
Tabla 2.

Livaluacion de lathoratorio de $S$ casos de intoxitación alcohistica en niñox menores do lo años

\begin{tabular}{|c|c|c|c|c|c|c|}
\hline Pasient: & & l & 2 & 3 & 4 & 5 \\
\hline Gaces Vemosom & $\begin{array}{l}\mathrm{pH} \\
\mathrm{PCO}_{2} \\
\mathrm{CO}_{2} \\
\mathrm{BF}\end{array}$ & $\begin{array}{l}7,13 \\
13,3 \\
12 \\
22\end{array}$ & $\begin{array}{c}7,47 \\
19,9 \\
14 \\
6\end{array}$ & & & $\begin{array}{l}7,34 \\
23 \\
13 \\
-10,3\end{array}$ \\
\hline Glicemin $\mathrm{g} / 1$ & & 0.58 & 0,9 & 0,30 & & 1,5 \\
\hline Bilirrubind mg/dl & & 0.9 & 0.7 & & & 0,5 \\
\hline $\begin{array}{l}\text { Transaminasas } \\
\text { [!III }\end{array}$ & $\begin{array}{l}\text { SGOT } \\
\text { SGPT }\end{array}$ & $\begin{array}{r}152 \\
37\end{array}$ & $\begin{array}{l}284 \\
119\end{array}$ & & & $\begin{array}{l}312 \\
150\end{array}$ \\
\hline $\begin{array}{l}\text { Fosfatasas } \\
\text { Alcalinas CliI } \\
\text { IN } 98 \cdot 279\end{array}$ & & 846 & 786 & & & 879 \\
\hline Amilasenia Luldal & & $387^{(1)}$ & 180 & & & \\
\hline Alootiolemia gil & & 0,64 & ()$^{(2)}$ & & $(--)^{(3)}$ & $(\cdot)^{(3)}$ \\
\hline$L C R$ & & Normal & Normai & Normal & Nomnal & Normal \\
\hline
\end{tabular}

(1) Alcanza valores rormales al $6^{\circ}$ día de cvoluciôn.

(2) Tomada al $2^{\circ} \mathrm{d}$ dí de coolución.

(3) Tomata al $3^{\circ}$ dia de cvolución.

monia y uno bronquitis). El estado de nutrición fue evaluado por tablas de Sempé, los pacientes 2, 3 y 5 eran eutróficos y los pacientes 1 y 4 desnutridos, I y II respectivamente.

Los exámenes de laboratorio demostraron, entre tres pacientes en que se realizó estudio de gases venosos, acidosis metabólica en dos. Dos niños tuvieron hipoglicemia, sólo uno de ellos sufrió convulsiones. En los tres en que se midió fosfatasas alcalinas y transaminasas las respectivas concentraciones séricas estaban alteradas, sin aumento de la bilirrubinemia; sólo uno de los nifros estudiados mostró aumento de la amilase. mia que tardó 6 días en normalizarse, sin evidencia clínica de lesión pancréatica. En el único paciente en que se nidió alcoholemia en las primeras horas desde su ingreso (6 horas), ésta resultó positiva. En otros dos casos el examen fue tomado al segundo y tercer día de evolución con resultados negativos. El estudio de líquido cefalorraquídeo (citoquímico y cultivo) efectuado en todos los menores fue normal (tabla 2).
La evolución de los niños ingresados con compromiso de conciencia fue hacia la recuperación de ésta, al tercer día en los mayores y al octavo día en el paciente de menor edad (2 a 4 meses). La recuperación del estado sicomotor de los niños entre 2 y 3 años fue lento, demorando hasta 2 y 3 semanas en recuperar sus destrezas motrices y de lenguaje previas.

No hubo mortalidad en este estudio.

\section{DISCUSION}

La incidencia de ingestión etílica en la población general chilena es alta. Pese a ser una manifestación más del mismo patrón cultural, motivado por estímulos sociales y ambientales, ${ }^{1}$ el consumo de alcohol en niños reviste caracteres diferentes. Es conocido el hecho que los factores que inducen el hábito alcohólico operan desde la infancia; ${ }^{2}$ lamentablemente pocos estudios nacionales $y$ extranjeros se han referido a este tema lo que impide conocer en forma más exacta $y$ 
actualizada sus aspectos epidemiológicos y clínicos.

Aún cuando la pequer̃a casuística presentada no pernite análisis estadístico, la intoxicación etílica es más frecuente en varones y en el medio rurat, lo que unido a la motivación de la ingesta sugiere que el estímulo social presiona más en estos grupos. La bebida más usada fue el vino, hecho que refleja el poder adquisitivo y las costumbres del medio de los pacientes. ${ }^{2}$

E] niño presenta riesgos mayores que el adulno cuando bebe alcohot. Desde el punto de vista somático las lcsiones agudas (gastrointestinal, pancreática, hepática y principalmente neurológica) pueden aparecer en forma nas precoz y con menores ingestas etilicas: a esto se suman los efectos a largo plazo ya conocidos. Además es necesario considerar los efectos en el desarrolto social, ausentisuno escolar y sus proyecciones futuras en el trabajo y la familia. ${ }^{2,9}$ creando lun cítculo vicioso en sus respectivos hijers. Los hijos de padres alcolólicos sutren atcultolismo on mayor frecuencia que los de padres abstemios, y beben mís bien por motivaciones sicupatoló-

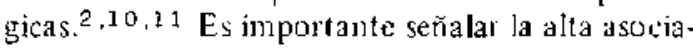
ción de drogadicción e ingestión excesiva de alcohol, la que estaría dada por la pertenencia a los mismos grupos vulnerables. ${ }^{10}$ Sin duda la prevención es el método más eficaz para evitar el uso del alcohol en los niños. Para elle se reco. mienda la educación sistematica en todos los niveles de enseñanza $y$ en las fuentes laborales,,$^{1,2}$ que debe ir unida con instrucción de la población general y los profesionales de salud. Estas acciones deben ir unidas a medidas de detección y referencia a centros de salud para su atención integral.

En todo niño con compromiso de conciencia de origen no precisado se debería medir alcohole. mia, y junto a esto, glicemia y gases arteriales que permitan un manejo adecuado de los niveles de glucosa y oxígeno a objeto de evitar las posibles secuelas originadas por sus alteraciones.

\section{RESUMEN}

Se analizan cinco casos de intoxicación alcohólica en menores de 10 años egresados del Hospital de Temuco durante 1984-1986. La mayoría de los pacientes son de sexo masculino, procedencia rural y la bebida fue ingerida con el consentimiento de los adultos responsables de ellos. En el cuadro clínico destaca el compromiso de conciencia, hipertonia, trismus y convulsiones, Las que gilo en un caso se relacionaron con hipoglicemia. La recuperación del estado sicomo. tor previo fue muy lenta en los menores de 3 años. Se comenta la necesidad de dar a conocet los peligros de la ingesta et ílica infantil y de tener presente el diagnóstico de intoxicación alcohólica atrte un paciente con compromiso de conciencia sith causa cvidente.

\section{REFERENCIAS}

1. Wontenzyo II.: Litado de Alcololismos en Chile. Cuadernos Médico Sociales Santiago. Chile 1980; 20: $11-17$.

2. Godoy, $C$., Montenegro $M$. Ugarte $G$.: Prevalencia del Hábito de Ingesta Al coló́fica en Escuelas Básicas del Area Central de Santiago, Rev Med Chile 197?; $105: 548-552$.

3. Astolf $k$. Higa $J$.: Intoxicación Alcohólica en la Infancia. [in: Meneghello J. ed. Pediatría, Santiago: Editorial Mediteráneo, $1985 ; 1477$.

4. Macfaren N.K.: Alcohol-Induced Ilypoglycemia in Childhood. Brit Med J 1970; L: 278-280.

5. Heggarty $H_{J}$.: Acute Alcoholic Hypoglycaemia in Two 4 - vear olds. Brit Med J 1970; 1: 280.

6. Fleisher $G_{\text {., ludwig }} S_{\text {. }}$. Toxicologic Emergencies En: Texbook of Pediatrics Emergency Medicine. Baltimore: Williams and wilkins, 1983; 503-506.

2. Malcolm J., Beriamín B. Sorbie A. Auchertoine $I$ : Alcohol Intoxication an underdiagnosed problem? Arch Dis Child 1985; 60: 762-763.

8. Ragan F., Somuets H., Hite S.: Ethanol ingestion in Children. JAMA 1979; 242: 2787-2788.

9. Dobert M.. Medina E.. Pavez E.: Programa de Prevención Prímaria del Alcoholismo en la Comunidad Escolar. Cuadernos Médico Sociales Santiago, Cliile $1980 ; 21: 4147$.

10. Cabrera F., Salomón C., López I., Vidal R.: Consumo de Bebidas Alcohólicas en la Población Escolar del Area Occidente de Santiago. Cuadernos Médico Sociales. Santjago, Chile $1982 ; 23: 5-12$.

11. Famularo $R$., Stone $K$., Popper Ch.: Preadolescent Alcohol Abuse and Dependence. Am J Psychiatry $1985 ; 142: 1187-1189$. 